\title{
A Method for Object Classification in Aerial/Satellite Images with Incorporating Geospatial Information
}

\author{
M. Popov ${ }^{1 *}$, M. Topolnytskyi ${ }^{1}$ and V. Pylypchuk ${ }^{2}$ \\ ${ }^{1}$ Scientific Centre for Aerospace Research of the Earth of the National Academy \\ of Sciences of Ukraine, Kyiv, Ukraine \\ ${ }^{2}$ Military-Diplomatic Academy named after Eugene Bereznyak, Kyiv, Ukraine
}

The manuscript was received on 11 February 2021 and was accepted after revision for publication as research paper 16 December 2021.

\begin{abstract}
:
Aerial and satellite multispectral images are important source of intelligence information. However, the object classification accuracy in those images for reasons such as camouflage, use of decoys, and others often turns out to be insufficient. The objective of the study is to develop a method for computer-aided analysis of aerial and satellite multispectral images, which allows improving classification accuracy. This objective is achieved by incorporating geospatial information (topographic, geodetic, about land cover types) into the classification process. As a mathematical basis of the method is used subjective logic of A. Jøsang. The effectiveness of the proposed method has been demonstrated by computer modeling using ArcGIS ModelBuilder tools.
\end{abstract}

\section{Keywords:}

cognitive bias, geospatial information, multispectral image, object classification, subjective logic, uncertainty

\section{Introduction}

It is well known that one of the richest sources of data on the terrain, landscape, operational environment, natural and man-made objects are aerial and satellite images [1]. The acquisition of such images and their analysis are the tasks of Imagery Intelligence activity [2]. Aerial and satellite images along with geospatial (topographic, structural morphological, geodetic, and other) data are widely used in Geospatial Intelligence [3, 4].

It should be noted that modern onboard cameras make it possible to form socalled digital multispectral images, consisting of a set of images, each of which dis-

\footnotetext{
* Corresponding author: Scientific Centre for Aerospace Research of the Earth of the National Academy of Sciences of Ukraine; Oles Honchar str., 55-b, Kyiv, UA-01054, Ukraine. Phone: +38044 48201 66, E-mail: mpopov@casre.kiev.ua. ORCID 0000-0003-1738-8227.
} 
plays surveyed territory in the separate enough narrow spectral zone of the optical radiation (visible or infrared) [5,6].

Analysis of the digital multispectral images is a cognitive process and it is performed by an operator-interpreter (O-I) [7]. Unfortunately, the cognitive process, due to its inherent complexity, is not straightforward and is accompanied by many difficulties [8]. To facilitate the work of the O-I, various expert and recommendation systems are developed [9-11]. The use of such systems allows to free the O-I from a significant part of the routine work, but all the work related to the semantic analysis (understanding) of the ground scene, including the classification of objects and the decisionmaking, nevertheless, are performed by the O-I.

In recent years, the difficulties of imagery interpretation are aggravated by the fact that an enemy uses camouflage and false targets more and more. In addition, the dynamics of military operations are increasing and the number of objects with a short lifetime is growing. Overall, these factors introduce additional uncertainty into the process of working with images and, therefore, the detection reliability and the classification accuracy of the objects are reduced. Experts see a way out of this situation in search of new paradigms for modeling and reducing uncertainty, developing effective methods of statistical and semantic fusion of data and information supplying different sources [12].

This study aims to develop a method to improve the accuracy of object classification in aerial and satellite multispectral images. Conceptually, taking also into account [12], the authors of the given paper go to the objective via reducing the degree of uncertainty inherent in the process of classifying objects by using contextual information. It should be noted that the concept of "context" includes a collection of relevant conditions such as space, time, environment, and surrounding influences that make a situation unique [13]. As stated in [14], "Context may provide information about the conditions of data and information acquisition, and it may constrain and influence the reasoning about objects and situations of interest."

In [15], a method is proposed in which contextual information is used to determine the operating modes of sensors that provide the most reliable and reactive decisions when targets are detected. The method is based on the provisions of the fuzzy set theory.

A supervised algorithm for image classification, which combines local and multiscale contextual information with rejection option, improving the classification performance, is described in [16]. As a probabilistic model for classification, a multinomial logistic regression is adopted.

In $[17,18]$, the attention was drawn to the fact that in cases where the objects of classification are located in the natural environment, it becomes possible to improve the classification performance by taking into account the properties of this environment. This phenomenon was demonstrated in [17], where the intellectualization of the classification process due to expert knowledge of the terrain made it possible to significantly improve the classification accuracy of such complex classes of objects, as different types of forest, agricultural areas, and pasturelands. In [18], it was shown how one can improve the accuracy of classification of remotely sensed imagery data by incorporating contextual elevation knowledge into the classification process. In [17] and [18], the apparatus of a Dempster-Schafer theory of evidence (DSTE) [19] was used as a mathematical platform.

A weakness of the studies marked above is that they do not fully take into account the specificity of image analysis in Geospatial Intelligence, which involves the 
prompt processing of large arrays of images under conditions of significant uncertainty and rigidly specified requirements for classification accuracy. Therefore, a new method for computer-aided analysis of aerial and satellite multispectral images using geospatial information is proposed, in the development of which the authors have tried to take into account the capacity of the modern geoinformation technologies.

Based on the fact that the accuracy of the final decision on the class of the object depends on the uncertainty degree of hypotheses, a quantitative criterion for uncertainty is introduced and a special procedure is proposed for reducing the uncertainty degree by sequentially incorporating geospatial context into the classification process.

A distinctive feature of the developed method is also the use of a special procedure of mitigation of the cognitive bias and errors of the O-I, which usually occur during the visual analysis of the digital multispectral image and the classification of the selected objects.

To assess the effectiveness of the proposed method, a computer modeling was carried out using ArcGIS ModelBuilder tools [20].

The rest of the paper is organized as follows. The methodological approach adopted in the study is described in Section 2. The basic concepts of subjective logic (SL) are presented in Section 3. Section 4 provides the description of the developed method as a main result of the study. Materials related to modeling the proposed method are presented in Section 5. Section 6 is dedicated to the discussion. Finally, Section 7 consists of the conclusion and directions for further work.

\section{Methodological Approach}

Automation of image analysis assumes a certain distribution of functions between the $\mathrm{O}-\mathrm{I}$ and the computer. We will proceed from the fact that human capabilities for detecting and classifying aerial or satellite images exceed the capabilities of today's digital algorithmic methods $[17,18]$. At the same time, a human's ability to make decisions in the presence of a large number of different factors is very limited. Based on these realities, it seems reasonable that the primary image analysis, including the detection of objects of interest and their classification, should be performed by the O-I, and then digital methods of data analysis and decision-making can be used to refine the results of cognitive classification.

Analyzing the image, the O-I puts forward different hypotheses about the possible classes of the objects under consideration. Attracting logic inference methods (induction, deduction, abduction et al), he/she estimates these hypotheses and considers the arguments pro and contra for each of them. The accuracy of their estimates is determined by two key factors.

The first factor is image quality. Quality of the images forming by the sensor is determined by its tactical and technical parameters, as well as the shooting conditions. The tactical and technical parameters of the sensor are relatively stable, but image quality can be significantly affected by changes in the transparency of the atmosphere, side flares, intrinsic noise of photodetectors, etc. These variations are stochastic and introduce so-called aleatory uncertainty into the process [21]. Reducing the level of aleatory uncertainty is possible, but for this, it is necessary to know the distribution functions of influencing parameters.

Another factor is the finiteness of knowledge inherent in any human. The lack of knowledge spawns so-called epistemic uncertainty [21, 22]. It has been experimentally proven $[23,24]$ that even the highly skilled interpreter working under uncertainties is 
prone to cognitive bias. Cognitive errors are committed by humans unconsciously and are manifested in a distorted perception of some image elements, unjustified regression shifts in estimates, unreasonable equalization of probabilities, etc.

The existing uncertainties, of course, negatively affect quality of the classification, and hence, the core idea of our methodological approach is that it is possible to improve the accuracy of the classification by reducing their level. There are two promising ways to reduce the impact of uncertainty factor on the results of object classification in aerial and satellite multispectral imagery.

Firstly, it is the attraction of geospatial contextual information. Here we proceed from the following considerations. The fact is that each class of military objects has the corresponding restrictions according to the conditions of the operation and the combat use, and these restrictions are determined by the tactical and technical specifications of objects of the concrete class. A significant part of the restrictions is related to the terrain, its characteristics, and state. Therefore, after analyzing the information about the given area, it is possible to establish the degree of "friendliness" (acceptable) of this area for objects of the certain class. The resulting assessment of the "friendliness" degree can be used to form a supplementary argument in favor of this or that decision regarding the class belonging of the object under consideration. In general, in order to reduce the uncertainty of the classification process, such elements of geospatial context as vegetation, physical relief of the terrain, swampiness, road network, and so on can be considered.

Secondly, if we understand the nature and possible consequences of the operator's cognitive bias, then we can envisage the possibility of their algorithmic correction (mitigation). In particular, one of the typical cognitive errors is that under conditions of a lack of information, the O-I assigns the same probability to all possible alternatives, i.e. they solve the classification problem by unreasonably equalizing the probabilities.

Finally, let us dwell on one more important issue. The implementation of a quantitative methodological approach requires a mathematical platform. The results obtained in $[17,18]$ indicate that the mathematical tool of DSTE can be used to classify objects under uncertainty.

However, as L. Zadeh demonstrated [25], the use of the DSTE methods in cases of conflicting hypotheses can lead to incorrect results. Also, it's worth noting that the use of the DSTE methods is connected with a significant computational burden.

For these reasons, authors of the given study propose to solve the problem of improving the accuracy of object classification in aerial and satellite multispectral images, relying on SL of A. Jøsang [26]. One of the advantages of the SL theory is that the description of any hypothesis in SL includes a quantitative assessment of the degree of its uncertainty [27]. Another important advantage is the simplicity of combining hypotheses, including the conflicting ones.

So, let us consider the basic provisions of SL.

\section{Basics of Subjective Logic}

$\mathrm{SL}$ is rooted into DSTE, so the terminology of both theories overlaps a lot. The subject of consideration in DSTE and SL is a system with a finite number of states (or classes) $K$. The states of the system are no longer decomposed, so they are called atomic ones. The states are mutually exclusive and exhaustive, i.e. at any time the system can be in only one of $K$ states. 
Every state of the system is presented by the corresponding hypothesis, so there will be $K$ atomic hypotheses, so-called singletons. Together, these hypothesessingletons form a set named A Frame of Discernment (FoD). The FoD set is usually marked by $\theta$ letter:

$$
\theta=\left\{H_{1}, \ldots, H_{k}, \ldots, H_{K}\right\}
$$

By combining the elements of FoD it is possible to build other, more numerous sets, which will contain all the singletons about the possible states of the system, all their possible combinations in the form of disjunctions of two, three terms, etc., as well as the $\theta$ set itself.

Full set constructed in this way is called A Power Set and is denoted as $2^{\theta}$ :

$$
2^{\theta}=\left\{\varnothing, H_{1}, \ldots, H_{k}, \ldots, H_{K}, H_{1} \cup H_{2}, H_{1} \cup H_{3}, \ldots, H_{1} \cup H_{2} \cup H_{3}, \ldots, \theta\right\}
$$

where a $\varnothing$ symbol denotes an empty set. Thus, a power set consists of $2^{K}$ elements.

Elements of the $2^{\theta}$ set, which through the disjunction unite two or more singletons, are called compound hypotheses. Every element $X$ of the $2^{\theta}$ set is associated with the number $m_{\theta}(X)$ complying the following conditions:

$$
\left\{\begin{array}{l}
m_{\theta}(X) \geq 0 \\
m_{\theta}(\varnothing)=0 \\
\sum_{X \in 2^{\theta}} m_{\theta}(X)=1
\end{array}\right.
$$

where $m_{\theta}(X)$ number is called a belief mass of the $X$ element. Elements of the $2^{\theta}$ set with zero belief mass are called focal ones. Note that for singletons of the power set, the concepts of mass of belief and Bayesian probability are identical.

The summation of the belief masses all elements of the $2^{\theta}$ set forms A Belief Mass Assignment (BMA).

For any element $X \mid X \in 2^{\theta}$ the following functions are introduced:

- belief

$$
b(X)=\sum_{Y \subseteq X} m_{\theta}(Y) ; X, Y \in 2^{\theta}
$$

- disbelief

$$
d(X)=\sum_{Y \cap X=\varnothing} m_{\theta}(Y) ; X, Y \in 2^{\theta}
$$

- uncertainty

$$
u(X)=\sum_{\substack{Y \cap X \neq \varnothing \\ Y \not \subset X}} m_{\theta}(Y) ; X, Y \in 2^{\theta}
$$

For any element $X \mid X \in 2^{\theta}$ it is true that:

$$
b(X)+d(X)+u(X)=1, \quad X \in 2^{\theta}, \quad X \neq \varnothing
$$

As noted above, the $2^{\theta}$ set contains both singletons and compounds. Let $X$ be an element of the $2^{\theta}$ set. Then the number of singletons that make up this element is called an atomicity and is denoted as $|X|$.

Using the concept of atomicity, one can estimate the magnitude of the possible overlap of the two elements of the $2^{\theta}$ set. Suppose $X, Y \in 2^{\theta}$, then for $Y \neq \varnothing$ the atomicity of element $X$ relative to element $Y$ is calculated as 


$$
a(X / Y)=\frac{|X \cap Y|}{|Y|}, \quad X, Y \in 2^{\theta}, \quad Y \neq \varnothing
$$

An index $a(X / Y)$ is called a relative atomicity. Note that $X \cap Y=\varnothing \Rightarrow a(X / Y)=0$ and $Y \subseteq X \Rightarrow a(X / Y)=1$. That is, the relative atomicity is characterized by a value between zero and one. Relative atomicity of the atomic hypothesis (singleton) to its FoD, denoted by $a(X / \theta)$, may be written as $a(X)$. If nothing else is specified, the relative atomicity of an element then refers to FoD.

$$
E(X)=\sum_{Y} m_{\theta}(Y) a(X / Y) ; X, Y \in 2^{\theta}
$$

The $E(X)$ function is the equivalent of a pignistic probability used in DSTE [28] and is a convenient criterion to evaluate uncertainty of the BMAs and the probability distributions. In the given study, we will utilize the $E(X)$ function to evaluate the uncertainty degree of the classification results.

Quite often, the researcher is not interested in all elements of the $\theta$ set, but only in some element $X \mid X \in 2^{\theta}$. In such cases, to simplify the calculations, it is proposed to convert initial multi-element set $\theta$ into a new $\tilde{\theta}^{X}$ set with the following properties:

- the $\tilde{\theta}^{X}$ set contains only two elements: $X$ and its addition $\neg X$,

- the BMA $m_{\tilde{\theta}^{X}}$ in the $\tilde{\theta}^{X}$ set is such that the values of the belief, disbelief, and uncertainty functions for $X$ element remain the same as they were in the initial set $\theta$, that's:

$$
\begin{aligned}
m_{\tilde{\theta}^{X}}(X) & =b(X) \\
m_{\tilde{\theta}^{X}}(\neg X) & =d(X) \\
m_{\tilde{\theta}^{X}}\left(\tilde{\theta}^{X}\right) & =u(X)
\end{aligned}
$$

A binary set $\tilde{\theta}^{X}$ with BMA $m_{\tilde{\theta}^{X}}$ satisfying (10), is called A Focused Frame of Discernment with focus on $X$. The focused relative atomicity of $X$ is expressed as follows:

$$
\begin{cases}a_{\tilde{\theta}^{X}}(X)=\frac{[E(X)-b(X)]}{u(X)} & \text { for } u(X) \neq 0 \\ a_{\tilde{\theta}^{X}}(X)=a(X) & \text { for } u(X)=0\end{cases}
$$

The operation of converting a multi-element set $\theta$ into a binary set $\tilde{\theta}^{X}$ plays an important role in situations when there are several different hypotheses according to the $X$ element and the problem of combining them arises. The atomic element $X$ of a binary set $\tilde{\theta}^{X}$ is called an opinion and is represented by a tuple

$$
w(X)=(b(X), d(X), u(X), a(X))
$$

where elements of the tuple are calculated by Eqs (4)-(6) and (8).

A significant advantage of the presentation (12) is that the uncertainty degree for the $X$ element is given here in an explicit form. In practice, several different opinions may exist regarding the same element, and then the problem of combining them arises.

This problem is solved in SL quite simply and elegantly. Suppose, there are two different opinions about the same element $X$ : 


$$
\begin{gathered}
w_{q}^{l}(X)=\left(b_{q}^{l}(X), d_{q}^{l}(X), u_{q}^{l}(X), a_{q}^{l}(X)\right) \\
w_{q}^{l+1}(X)=\left(b_{q}^{l+1}(X), d_{q}^{l+1}(X), u_{q}^{l+1}(X), a_{q}^{l+1}(X)\right)
\end{gathered}
$$

Then the combination of these two opinions can be written as the tuple

$$
w_{q}^{l, l+1}(X)=\left(b_{q}^{l, l+1}(X), d_{q}^{l, l+1}(X), u_{q}^{l, l+1}(X), a_{q}^{l, l+1}(X)\right)
$$

elements of which are calculated as follows:

$$
\begin{gathered}
b_{q}^{l, l+1}(X)=\frac{b_{q}^{l}(X) u_{q}^{l+1}(X)+b_{q}^{l+1}(X) u_{q}^{l}(X)}{u_{q}^{l}(X)+u_{q}^{l+1}(X)-u_{q}^{l}(X) u_{q}^{l+1}(X)} \\
d_{q}^{l, l+1}(X)=\frac{d_{q}^{l}(X) u_{q}^{l+1}(X)+d_{q}^{l+1}(X) u_{q}^{l}(X)}{u_{q}^{l}(X)+u_{q}^{l+1}(X)-u_{q}^{l}(X) u_{q}^{l+1}(X)} \\
u_{q}^{l, l+1}(X)=\frac{u_{q}^{l}(X) u_{q}^{l+1}(X)}{u_{q}^{l}(X)+u_{q}^{l+1}(X)-u_{q}^{l}(X) u_{q}^{l+1}(X)} \\
a_{q}^{l, l+1}(X)=\frac{a_{q}^{l}(X) u_{q}^{l+1}(X)+a_{q}^{l+1}(X) u_{q}^{l}(X)-\left(a_{q}^{l}(X)+a_{q}^{l+1}(X)\right) u_{q}^{l}(X) u_{q}^{l+1}(X)}{u_{q}^{l}(X)+u_{q}^{l+1}(X)-2 u_{q}^{l}(X) u_{q}^{l+1}(X)}
\end{gathered}
$$

The tuple (14), whose elements are calculated with help of Eq. (15), is called $a$ consensus [29].

So, now we have the opportunity to present a core result of our study - a new method for computer-aided analysis of aerial and satellite multispectral images.

\section{Results}

Suppose the digital multispectral image to be analyzed consists of the $L$ zonal images. The procedural scheme of the proposed method is shown in Fig. 1.

The proposed method consists of 10 steps. Let us move on to describing their content.

\subsection{Digital Image Processing}

The digital processing procedure itself includes radiometric and geometric correction of each of $L$ "raw" zonal images, as well as their georeferencing (what corresponds to 2A Level of the image processing [30]). As a rule, the corrected and georeferenced zonal images are presented in the GeoTIFF format.

If the external conditions that existed at the moment when the image was formed (for example, the state of the atmosphere) are known, then at this step, a posteriori image processing can also be carried out. Such processing will help reduce aleatory uncertainty.

We emphasize that all computations are conducted under the guidance of the O-I.

\subsection{Visual Analysis of Digital Image}

The O-I studies the multispectral image in a visual way, selects objects of interest within each of the zonal images, and determines their coordinates. 


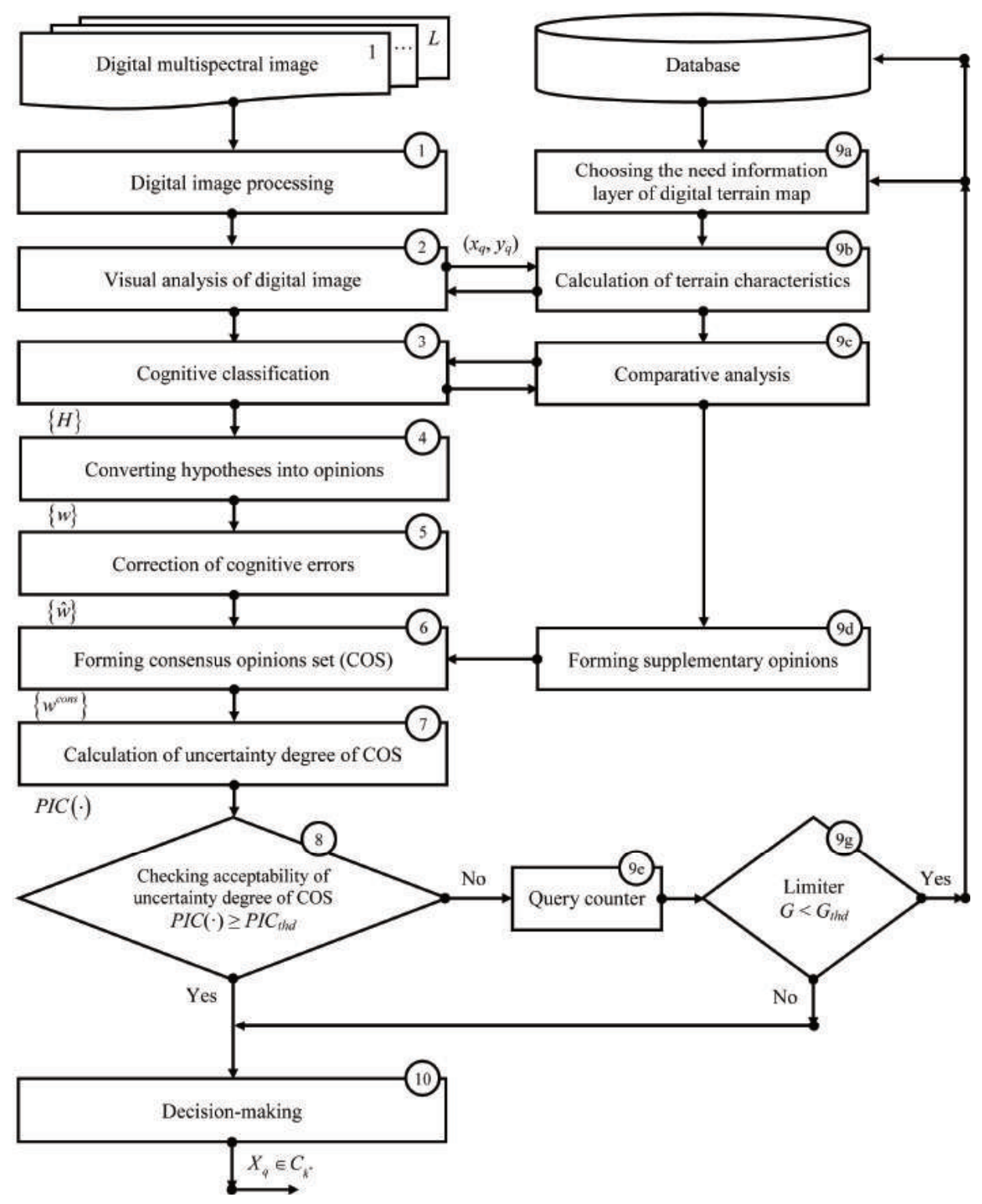

Fig. 1 Procedural scheme of the proposed method

\subsection{Cognitive Classification}

At this step, the O-I performs the visual (cognitive) classification of the selected objects.

Assume the O-I has concluded that the image under consideration contains $Q$ objects belonging to $K$ different classes. For $K$ classes, there will accordingly be $K$ different hypotheses $H$, and the set of these hypotheses can be considered as FoD 


$$
\theta=\left\{H_{1}, \ldots, H_{k}, \ldots, H_{K}\right\}, k=1,2, \ldots, K
$$

From the elements of this set, one can compose a power set. For example, if $K=3$, then the power set will look as follows:

$$
2^{\theta}=\left\{\varnothing, H_{1}, H_{2}, H_{3}, H_{12}, H_{13}, H_{123}\right\}
$$

where $H_{12}=H_{1} \cup H_{2}, H_{13}=H_{1} \cup H_{3}, H_{23}=H_{2} \cup H_{3}, H_{123}=H_{1} \cup H_{2} \cup H_{3}$

Each element $H_{j}$ into the $2^{\theta}$ power set has its own belief mass $m_{j}$. This fact is written as a pair $\left(H_{j}, m_{j}\right)$. So, the O-I gives out the cognitive classification result for an arbitrary object $X_{q}$ in the $l$-th zonal image in the following form:

$$
R^{l}\left(X_{q}\right)=\left\{\left\langle H_{1}^{l}\left(X_{q}\right), m_{1}^{l}\right\rangle, \ldots,\left\langle H_{j}^{l}\left(X_{q}\right), m_{j}^{l}\right\rangle, \ldots,\left\langle H_{J}^{l}\left(X_{q}\right), m_{J}^{l}\right\rangle\right\}
$$

where $H_{j}^{l}\left(X_{q}\right)$ - the hypothesis according class belonging of the $X_{q}$ object; $m_{j}^{l}-$ the belief mass for the $H_{j}^{l}\left(X_{q}\right)$ hypothesis; $J=2^{K}-1 ; l=1,2, \ldots, L ; q=1,2, \ldots, Q$.

In total, for each object of interest, the O-I gives out $L$ results regarding its possible class belonging in a form (18). Each such a result is the set of hypotheses, which can contain from 1 to $K$ focal elements. However, SL manipulates not by hypotheses, but by opinions; so, the next step is required.

\subsection{Converting Hypotheses into Opinions}

Some hypotheses of FoD can be presented within the $2^{\theta}$ power set (17) not only once, but they can also be presented here as a disjunctive element of compound hypotheses. We need to obtain the BMA for a set whose elements will be only singletons.

In addition, it should take into account that the transition from the initial set $\theta$ to the binary set $\tilde{\theta}^{H}$ is complicated by the presence of an interdependence between the expected probability $E\left(H_{k}^{l}\right)$ of each hypothesis (Eq. (9)) and relative atomicity $a_{\tilde{\theta}^{X}}\left(H_{k}^{l}\right)$ (Eq. (11)). This interdependence can be eliminated with the procedure of the hierarchical probability transformation, proposed in [31]. Its essence is as follows.

Suppose there are FoD $\theta=\left\{H_{1}^{l}, H_{2}^{l}, H_{3}^{l}\right\}$ and the power set including both atomic and compound hypotheses:

$$
2^{\theta}=\left\{\begin{array}{l}
\left\langle H_{1}^{l}, m_{1}^{l}\right\rangle,\left\langle H_{2}^{l}, m_{2}^{l}\right\rangle,\left\langle H_{3}^{l}, m_{3}^{l}\right\rangle,\left\langle H_{12}^{l}, m_{12}^{l}\right\rangle, \\
\left\langle H_{13}^{l}, m_{13}^{l}\right\rangle,\left\langle H_{23}^{l}, m_{23}^{l}\right\rangle,\left\langle H_{123}^{l}, m_{123}^{l}\right\rangle
\end{array}\right\}
$$

Each of the hypotheses $H_{n}^{l}$ has a corresponding belief mass $m_{n}^{l}$ (here $n=1,2,3,4,5,6,7)$. Then the procedure of hierarchical probability transformation can be explained with help of Fig. 2.

The transforming operation begins with the reallocation of the mass of the threeterm hypothesis $\left\langle H_{123}^{l}, m_{123}^{l}\right\rangle \equiv\left\langle H_{1}^{l} \cup H_{2}^{l} \cup H_{3}^{l}, m_{123}^{l}\right\rangle$ to the masses of the two-term sets $\left\langle H_{1}^{l} \cup H_{2}^{l}, m_{12}^{l}\right\rangle,\left\langle H_{1}^{l} \cup H_{3}^{l}, m_{13}^{l}\right\rangle,\left\langle H_{2}^{l} \cup H_{3}^{l}, m_{23}^{l}\right\rangle$.

This stage occurs by adding to their masses the product of the mass $m_{123}^{l}$ of the compound hypothesis $H_{123}^{l}$ by a reallocation factor that characterizes the proportion of the corresponding mass in the total masses $H_{12}^{l}, H_{13}^{l}$, and $H_{23}^{l}$.

In the second stage, the two-term hypotheses-disjunctions are decomposed in the singletons $H_{1}^{l}, H_{2}^{l}$, and $H_{3}^{l}$, and for each of them mass is determined in the same way. The reallocation of masses between the hypotheses at these stages is carried out in accordance with the expression: 


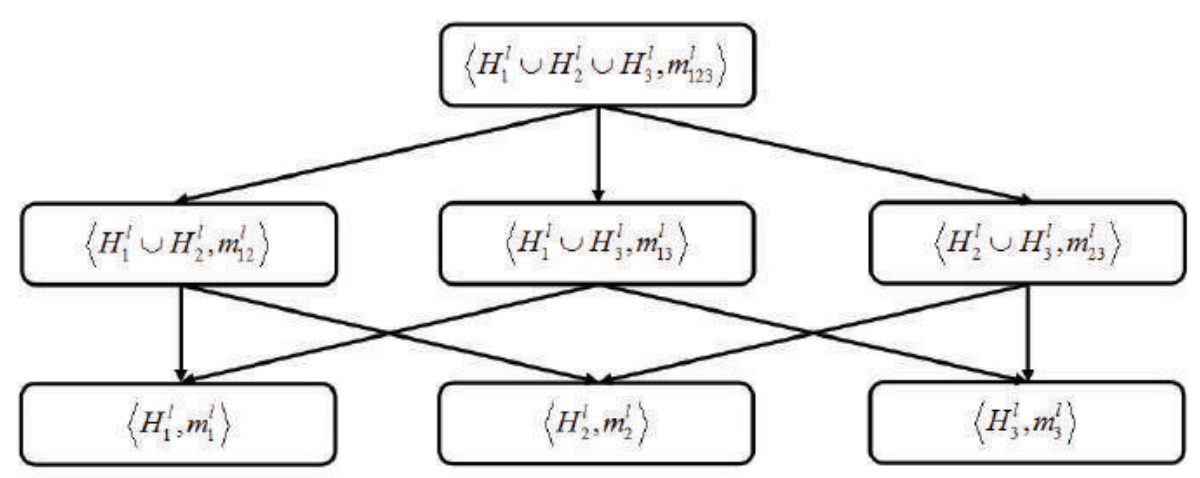

Fig. 2 Explanation of hierarchical probability transformation procedure

$$
E_{H T}(H(\lambda-1))=m() H(\lambda-1)+\sum_{\substack{H(\lambda) \supset H(\lambda-1) \\ H(\lambda), H(\lambda-1) \in 2^{\theta}}}[m(H(\lambda)) R(H(\lambda-1), H(\lambda))]
$$

where $R(H(\lambda-1), H(\lambda))$ is the proportional reallocation factor, which is calculated in the following way:

$$
R(H(\lambda-1), H(\lambda))=\frac{m(H(\lambda-1))}{\sum_{\substack{H(\lambda) \supset H(\lambda-1) \\ H(\lambda), H(\lambda-1) \in 2^{\theta}}} m(H(\lambda-1))}
$$

where $\lambda$ is the transformation stage.

Therefore, generally, the result of the application of the hierarchical probability transformation procedure will be the decomposition of an initial set of $N$ atomic and compound hypotheses only atomic hypotheses (singletons) with determination of the probability (mass) of each hypothesis.

Thus, taking into account Eqs (4)-(6), (11), (20), and (21), on the basis of the hypotheses defined in step 3, belief $b(\cdot)$, disbelief $d(\cdot)$, and uncertainty $u(\cdot)$, expected probability $E(\cdot)$, and relative atomicity $a_{\tilde{\theta}^{X}}(\cdot)$ can be calculated, as well as the relevant opinions can be formed.

If now we will rely on the probability distributions of the hypotheses that have been by the O-I within a framework of a zonal image in study, then the described above procedure of hierarchical probability transformation allows one to obtain the opinion regarding the class belonging of each selected object:

$$
H_{k}^{l} \Leftrightarrow w_{k}^{l}\left(b_{k}^{l}, d_{k}^{l}, u_{k}^{l}, a_{k}^{l}\right), l=1,2, \ldots, L, k=1,2, \ldots, K
$$

\subsection{Correction of Cognitive Errors}

As pointed out in Section 2, one of the factors limiting classification accuracy is cognitive bias of the O-I. However, the methodology of our study assumes the possibility of algorithmic correction of cognitive errors. Our method provides for algorithmic correction (mitigation) of a cognitive error that occurs when, due to a lack of information, the operator unreasonably levels the probabilities of hypotheses. 
Let us introduce the necessary explanations. Suppose the task is to establish whether the object under consideration can belong to some class $c$, but there is no information about this object. The usual practice in such cases is that the O-I makes an equiprobable conclusion about the belonging / non-belonging of the given object to the specified class. In accordance with categories of SL, this conclusion is expressed through the corresponding opinion

$$
w_{c}\left(b_{c}, d_{c}, u_{c}\right)=\left(0.5,0.5, u_{c}\right)
$$

If to accept the condition (7), then the value of $u_{c}$ into (23) should be equal to zero, but this contradicts the reality (the initial uncertainty has not disappeared anywhere). Thus, making the equiprobable conclusion, the O-I commits a cognitive error. To correct this error, a procedure for maximizing uncertainty [32] is proposed. Through this procedure, the opinion $w(X)=(b(X), d(X), u(X), a(X))$ is transformed into an opinion $\hat{w}(X)=(\hat{b}(X), \hat{d}(X), \hat{u}(X), \hat{a}(X))$ in which uncertainty that occurs due to the lack of information is maximally reflected.

The procedure is performed according to the formulae: if $E(X) \leq a(X)$ then

$$
\left\{\begin{array}{l}
\hat{b}(X)=0 \\
\hat{d}(X)=1-u(X)-\frac{b(X)}{a(X)} \\
\hat{u}(X)=u(X)+\frac{b(X)}{a(X)} \\
\hat{a}(X)=a(X)
\end{array}\right.
$$

if $E(X)>a(X)$ then

$$
\left\{\begin{array}{l}
\hat{b}(X)=1-u(X)-\frac{d(X)}{1-a(X)} \\
\hat{d}(X)=0 \\
\hat{u}(X)=u(X)+\frac{d(X)}{1-a(X)} \\
\hat{a}(X)=a(X)
\end{array}\right.
$$

Thus, for any $q$-th selected object $(q=1,2, \ldots, Q), L$ tuples are formed, each of which consists of $K$ independent opinions regarding their possible class belonging:

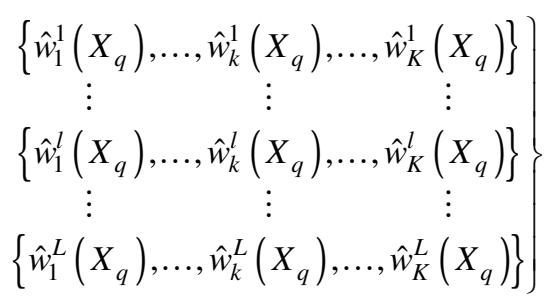

The next task is to unite (combine) these opinions and thus obtain a set of consensus opinions. 


\subsection{Forming Consensus Opinions Set (COS)}

A process of combining opinions from Eq. (25) separately for each of classes is conducted using Eqs (13)-(15). The process starts from combining $\hat{w}_{k}^{1}\left(X_{q}\right)$ and $\hat{w}_{k}^{2}\left(X_{q}\right)$ opinions. After that, the $\hat{w}_{k}^{1,2}\left(X_{q}\right)$ result is combined with the $\hat{w}_{k}^{3}\left(X_{q}\right)$ opinion and so on. Upon completion of $(L-1)$ combining operations, the $w_{k}^{\text {cons }}\left(X_{q}\right)$ consensus opinion is formed for the $q$-th object regarding its belonging to the $k$ class.

In a similar way, the consensus opinions are formed about belonging of the object under consideration to each of the remaining $(K-1)$ classes and, thus, the COS is formed for the $q$-th object:

$$
\left\{w_{1}^{\text {cons }}\left(X_{q}\right), \ldots, w_{k}^{\text {cons }}\left(X_{q}\right), \ldots, w_{K}^{\text {cons }}\left(X_{q}\right)\right\}
$$

The fact that the COS (26) contains not one but $K$ alternatives for the class of the object in question means that there is inherent epistemic uncertainty. Since the quality of decision on the object class belonging depends directly on the degree of the existing uncertainty, it is necessary to get the estimation of this degree.

\subsection{Calculation of the Uncertainty Degree of COS}

Consider how this step is performed on the example of the $X_{q}$ object with the COS (26). Knowing the set (26) for $q$-th object allows us to calculate the distribution of the expected probabilities by Eq. (9):

$$
E\left(X_{q}\right)=\left\{E_{1}\left(X_{q}\right), \ldots, E_{k}\left(X_{q}\right), \ldots, E_{K}\left(X_{q}\right)\right\}
$$

To estimate the uncertainty of the distribution of expected probabilities (27), we use A Probability Information Content Index (PIC) introduced by J. Sudano [33]:

$$
\operatorname{PIC}\left[E\left(X_{q}\right)\right]=1+\frac{\sum_{k=1}^{K} E_{k}\left(X_{q}\right) \cdot \log _{2}\left(E_{k}\left(X_{q}\right)\right)}{\log _{2} K}
$$

A PIC value of zero indicates that all the opinions have the same expected probability value and so it is not possible to classify the object. This is a case of full ignorance (i.e. of maximal uncertainty). A PIC value of one indicates that an expected probability value of one has one opinion only, i.e. in this case, there is no uncertainty. Thus, the PIC is a simple and convenient index for estimating the uncertainty degree of the consensus opinions.

\subsection{Checking Acceptability of the Uncertainty Degree of COS}

Let the $\operatorname{COS}\left\{w_{1}^{\text {cons }}\left(X_{q}\right), \ldots, w_{k}^{\text {cons }}\left(X_{q}\right), \ldots, w_{K}^{\text {cons }}\left(X_{q}\right)\right\}$ be characterized by the distribution of the expected probabilities $E^{\text {cons }}\left(X_{q}\right)=\left\{E_{1}^{\text {cons }}\left(X_{q}\right), \ldots, E_{k}^{\text {cons }}\left(X_{q}\right), \ldots, E_{K}^{\text {cons }}\left(X_{q}\right)\right\}$. The uncertainty degree of specified COS will be estimated using $\operatorname{PIC}\left[E^{\text {cons }}\left(X_{q}\right)\right]$. Then the criterion of acceptability of the uncertainty degree of the COS can be written by inequality:

$$
P I C\left[E^{\text {cons }}\left(X_{q}\right)\right] \geq P I C_{\text {thd }}
$$

where $P I C_{\text {thd }}$ is the threshold. 
If inequality (29) is held, then this fact indicates that the uncertainty degree of the $\operatorname{COS}$ (26) allows one to make a cogent decision on the class of the $X_{q}$ object. Our experience shows that the $P I C_{\text {thd }}$ value should be about 0.5 , not less.

Failure to meet inequality (29) indicates high degree of the epistemological uncertainty which may be reduced by attracting ("pulling up") geospatial context (geomorphological, hydrographic, landscape information, digital terrain maps, digital elevation model, and so on). The required information is stored in a database.

\subsection{Forming Supplementary Opinions}

The proposed approach is based on the assumption that it is possible to improve the accuracy of object classification in aerial and satellite images by incorporating geospatial information (information about the terrain) into the classification process.

The fact is that each class of military objects has the corresponding restrictions according to the conditions of the operation and the combat use, and these restrictions are determined by the tactical and technical specifications of the objects of a class. A significant part of these restrictions is related to the surrounding terrain, its characteristics, and state. Therefore, after analyzing the information about the characteristics of the given area, it is possible to establish how "friendly" (acceptable) this area is for objects of the certain class. Based on the results of such an analysis, supplementary opinions can be formed and introduced into the decision-making process about the class of the object under study.

As stated above, the need for supplementary opinions arises in cases where inequality (29) does not hold. Let us consider the procedure of forming the supplementary opinion on the following example. Assume, the O-I selected some object $X_{q}$ in two different zonal images of the same area of terrain at the point with coordinates $\left(x_{q}, y_{q}\right)$ and conjectures that the specified object can be either a Tank $\left(H_{1}\right.$ hypothesis $)$ or an Infantry Fighting Vehicle (IFV) ( $H_{2}$ hypothesis).

Suppose visual analysis of the first zonal image led to the following result:

$$
\left.\left\{\left\langle H_{1}^{1}, 0.6\right\rangle,\left\langle H_{2}^{1}, 0.1\right\rangle,\left\langle H_{1}^{1} \cup H_{2}^{1}, 0.3\right\rangle\right\}\right|_{\left(x_{q}, y_{q}\right)}
$$

and visual analysis of the second zonal image yielded the following result:

$$
\left.\left\{\left\langle H_{1}^{2}, 0.2\right\rangle,\left\langle H_{2}^{2}, 0.6\right\rangle,\left\langle H_{1}^{2} \cup H_{2}^{2}, 0.2\right\rangle\right\}\right|_{\left(x_{q}, y_{q}\right)}
$$

Having hypotheses (30), it is not difficult to go to the opinions: $w^{1}($ Tank $)=(0.6,0.1,0.3,0.5), w^{1}(\mathrm{IFV})=(0.1,0.6,0.3,0.5)$, $w^{2}($ Tank $)=(0.2,0.6,0.2,0.5)$ and $w^{2}(\mathrm{IFV})=(0.6,0.2,0.2,0.5)$.

After that, using the Eq. (24), we correct the cognitive error of the O-I and then have: $\hat{w}^{1}($ Tank $)=(0.5,0.0,0.5,0.5), \hat{w}^{1}(\operatorname{IFV})=(0.0,0.5,0.5,0.5)$, $\hat{w}^{2}($ Tank $)=(0.0,0.4,0.6,0.5)$ and $\hat{w}^{2}(\operatorname{IFV})=(0.4,0.0,0.6,0.5)$.

Combining these opinions and applying the Eq. (15), we obtain the pair of the consensus opinions:

$$
w^{\text {cons }}(\text { Tank })=(0.38,0.25,0.38,0.50), w^{\text {cons }}(\text { IFV })=(0.25,0.38,0.38,0.50)
$$

Having the consensus opinions (31), we calculate the expected probability $E^{\text {cons }}(\cdot)$ for the opinions "Tank" and "IFV": 


$$
\left\{E^{\text {cons }}(\text { Tank })=0.56, E^{\text {cons }}(\text { IFV })=0.44\right\}
$$

According to the procedural scheme of the method (see Fig. 1), it is necessary to assess the uncertainty degree of the pair of consensus opinions (30). To do this, we substitute the values (32) of the expected probability for the "Tank" and the "IFV" opinions into the Eq. (28) and get $P I C\left[E^{\text {cons }}\left(X_{q}\right)\right]=0.01$.

If to take $P I C_{\text {thd }}=0.5$, inequality (29) for the pair (31) does not hold. This fact indicates that the specified set by its uncertainty degree is not able to ensure a cogent decision on the class of the object. Therefore, supplementary information is needed to reduce the level of existing uncertainty.

Let see how to get such information using the following example. It is known that a Tank and an IFV have different hill climb angles. As a rule, for tanks, a hill climb angle is usually about $30^{\circ}$, and for IFVs this angle is slightly larger, about $35^{\circ}$. This difference between climb angles can be used to form a supplementary opinion.

Knowing the coordinates $\left(x_{q}, y_{q}\right)$ of the selected object $X_{q}$, the O-I requests the digital map of the area under study from the database selects the layer of terrain relief and receives information about the relief steepness value. Suppose the steepness value within this area turned out to be $32^{\circ}$. Then, comparing the relief steepness angle with the hill climb angles, one can more accurately establish class of object in question.

Let $\beta$ is a relief steepness within the area of location of the $X_{q}$ object and $\gamma_{t}$ and $\gamma_{v}$ are hill climb angles of the Tank and the IFV correspondingly. Then support for hypothesis $H_{1}$ ( $X_{q}$ object is Tank) may be expressed by the probability

$$
P\left(H_{1}\right)=\left\{\begin{array}{l}
0.95, \text { if }\left(\gamma_{t}-\beta\right)>0 \\
0, \text { otherwise }
\end{array}\right.
$$

and the support for the $H_{2}$ hypothesis ( $X_{q}$ object is IFV) may be written as the probability

$$
P\left(H_{2}\right)=\left\{\begin{array}{l}
0.95, \text { if }\left(\gamma_{v}-\beta\right)>0 \\
0, \text { otherwise }
\end{array}\right.
$$

Moving from the hypotheses (33) and (34) to the opinions, we get argumentsopinions according to which the $X_{q}$ object is either the Tank:

$$
w^{\text {sup }}\left(X_{q} \text { is Tank }\right)=w^{\text {sup }}(\text { Tank })=(0,0.95,0.05,0.5)
$$

or the IFV:

$$
w^{\text {sup }}\left(X_{q} \text { is IFV }\right)=w^{\text {sup }}(\mathrm{IFV})=(0.95,0,0.05,0.5)
$$

The opinions (35) and (36) may be joined into the tuple:

$$
\left\{w^{\text {sup }}(\text { Tank }), w^{\text {sup }}(\text { IFV })\right\}
$$

Considering the supplementary opinions from the tuple (36) and the consensus opinions (31) together, and omitting the calculations foreseen at the step 6 , we can formulate the opinions pair:

$$
\{w(\text { Tank }), w(\text { IFV })\}
$$

The expected probability for each of the two hypotheses into the opinions set (37) will have the following values: $E($ Tank $)=0.07, E($ IFV $)=0.93$. Substituting these 
values in the Eq. (29), we find: PIC $=0.64$. Comparing this value with $P I C_{\mathrm{thd}}=0.5$, one can conclude that the uncertainty degree of the opinions pair (38) formed with the involvement of supplementary information allows one to proceed to the final step 10.

If the given supplementary opinion including in the COS did not ensure the satisfaction of inequality (29), the O-I queries the database in order to form the next supplementary opinion, and so on. As soon as inequality (29) is satisfied, the process of COS forming ceases, after which the final decision on the class belonging of the object under consideration is made.

However, there may be situations when the uncertainty of the consensus set does not decrease (inequality (29) does not hold) with the increase of the number of supplementary opinions. For similar cases, a stop mechanism is foreseen which is implemented using a query counter and a limiter (see Fig. 1). The counter registers all queries of the O-I to the DB, and their total number $G$ is compared with the $G_{\text {thd }}$ threshold. If $G \leq G_{\text {thd }}$, then the current query is made to the database and a new supplementary opinion is formed. If the $G$ number of the formed supplementary opinions has exceeded $G_{\text {thd }}$ number, then the transition is carried out to the procedure of final decision-making regarding the class of the $X_{q}$ object under consideration.

\subsection{Decision-Making}

Assume there is a full set of classes $C=\left\{c_{1}, \ldots, c_{k}, \ldots, c_{K}\right\}$ and a set of $K$ consensus opinions

$$
\left\{w_{1}^{\text {cons }}\left(X_{q}\right), \ldots, w_{k}^{\text {cons }}\left(X_{q}\right), \ldots, w_{K}^{\text {cons }}\left(X_{q}\right)\right\}
$$

regarding possible classes of some object $X_{q}$. It is also assumed that the distribution of the expected probabilities $\left\{E_{1}^{\text {cons }}\left(X_{q}\right), \ldots, E_{k}^{\text {cons }}\left(X_{q}\right), \ldots, E_{K}^{\text {cons }}\left(X_{q}\right)\right\}$ of these opinions is known.

Then the final decision on the class belonging of the $X_{q}$ object is determined by that opinion from (39), which has the highest value of the expected probability.

Formally, this decision rule is noted as follows: the object $X_{q}$ under consideration refers to the $c_{k^{*}}$ class $\left(c_{k^{*}} \subset C\right)$, the label of which is defined as

$$
k^{*}=\operatorname{subscript}\left\{\max _{k=1,2, \cdots, K}\left[E_{k}^{\text {cons }}\left(X_{q}\right)\right]\right\}
$$

We emphasize once more that the steps from the third to the tenth are performed separately and independently for each of the $X_{q}$ objects $(q=1,2, \ldots, Q)$ which have been selected by the O-I at the second step.

\section{Modeling}

The performance of the proposed method was evaluated by modeling using simulated multispectral image compiled from 4 zonal mages in raster format. The image consists of a scene including 32 objects of three different classes - tanks, light armored multipurpose towing vehicles, and armored personnel carriers. The modeling was carried out leveraging the ESRI ArcGIS.

The multispectral image was presented to the highly skilled O-I for the visual analysis. Analyzing this image, the O-I has selected all 32 objects and determined the 
coordinates of each of them. The classification of each zonal image was performed by the O-I separately.

The result of the cognitive classification of each object $X_{q}$ into each of the $L$ zonal images was expressed in the following form:

$$
R^{l}\left(X_{q}\right)=\left\{\left\langle H_{1}^{l}\left(X_{q}\right), m_{1}^{l}\right\rangle, \ldots,\left\langle H_{j}^{l}\left(X_{q}\right), m_{j}^{l}\right\rangle, \ldots,\left\langle H_{J}^{l}\left(X_{q}\right), m_{J}^{l}\right\rangle\right\}
$$

Unfortunately, in general case among hypotheses within (41), there can be not only atomic but also compound hypotheses, and this fact complicates obtaining an unambiguous decision on the class belonging of the objects under consideration. However, the problem can be solved using the concept of pignistic probability introduced in [28].

The pignistic probability for any $H_{k}$ hypothesis is calculated as follows:

$$
\operatorname{BetP}\left(H_{k}\right)=\sum_{\substack{H_{k} \in H_{j} \\ H_{j} \in 2^{\theta}}} \frac{m\left(H_{j}\right)}{\left|H_{j}\right|} ; k=1,2, \ldots, K
$$

where $\left|H_{j}\right|$ - the cardinal number of the $H_{j}$ set.

Then the class of any object $X_{q}(q=1,2, \ldots, Q)$ is determined by the hypothesis within (41), which has the highest value of the pignistic probability.

In this way, the cognitive classification of each zonal image was carried out and the corresponding decisions were made regarding each of the 32 objects. The probability of correct classification of the objects separately for each zonal image of the multispectral image is given in Tab. 1.

Tab. 1 Probabilities of correct classification

\begin{tabular}{|c|c|}
\hline Zonal image $(l)$ & Probability of correct classification \\
\hline$l=1$ & 0.61 \\
\hline$l=2$ & 0.64 \\
\hline$l=3$ & 0.63 \\
\hline$l=L=4$ & 0.72 \\
\hline
\end{tabular}

Thus, the probability of the correct classification of objects in the digital multispectral image under study was, on the whole, 0.65 .

Further work aimed at improving the classification accuracy was carried out using a computer model built in the ESRI ArcGIS.

Input data for the computer model are:

- the results of cognitive (visual) interpretation of the digital multispectral image in the vector format,

- the raster layers, depicting the earth's surface (ES) and the terrain relief (RT), and the vector layer portraying the road network (RN).

To model the proposed method, a SLTToolbox toolkit (Fig. 3) was developed in the ArcGIS ModelBuilder (ArcGIS Desktop Advanced 10.7). The ArcGIS Spatial Analyst for Desktop 10.7 module was additionally used, as well as the LUPTool.py script on the Python.

The SLTToolbox toolkit consists of four tools: LUPTool, LUP\&FirstSupplementOpinion, LUP\&TwoSupplementOpinions, and LUP\&ThreeSupplementOpinions. 


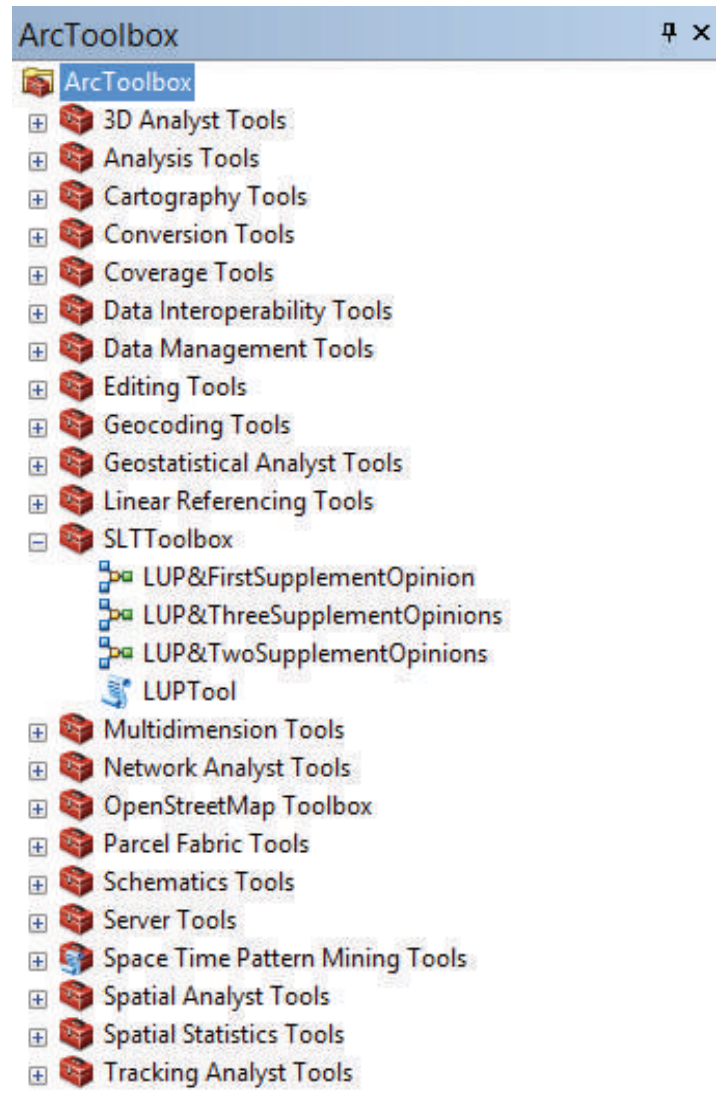

Fig. 3 SLTToolbox toolkit

The LUPTool tool is built on the basis of the LUPTool.py script and has the purpose to form the consensus opinion based on data sources that are represented in the vector format. In our case, such sources may be:

- the results of cognitive (visual) interpretation of the 4 zonal images of the digital multispectral image,

- the consensus opinion (Positions) formed by the way combining the results of visual interpretation of the 4 zonal images, and geospatial information about the terrain characteristics (LC, RT, and RN).

The LUP\&FirstSupplementOpinion tool is intended to form the consensus opinions using:

- the consensus opinion (Positions) formed on the base of the results of the visual interpretation of the digital multispectral image,

- geospatial information related to any one of 3 context elements: LC or RT or RN.

The LUP\&TwoSupplementOpinions tool is intended to form the consensus opinions using:

- the consensus opinion (Positions) formed on the base of the results of the visual interpretation of the digital multispectral image, 
- geospatial information related to any pair of 3 context elements: (LC \& RT) or (LC \& RN) or (RT \& RN).

The LUP\&ThreeSupplementOpinions tool is intended to form the consensus opinions using:

- the consensus opinion (Positions) formed on the base of the results of the visual interpretation of the digital multispectral image,

- geospatial information related to 3 context elements: ES \& TR \& RN.

The implementation of the LUP\&ThreeSupplementOpinions tool in the ArcGIS ModelBuilder is shown in Fig. 4. Further on, the computer model was published using ArcGIS Server 10.7 as an asynchronous geoprocessing service that was integrated into Portal for ArcGIS 10.7 (Fig. 5). In addition, the input data (Positions, ES, RT and RN) were published separately as a map service. For this service, the map cache is not created. Raster data are presented in GRID format, vector data are presented in SHP format.

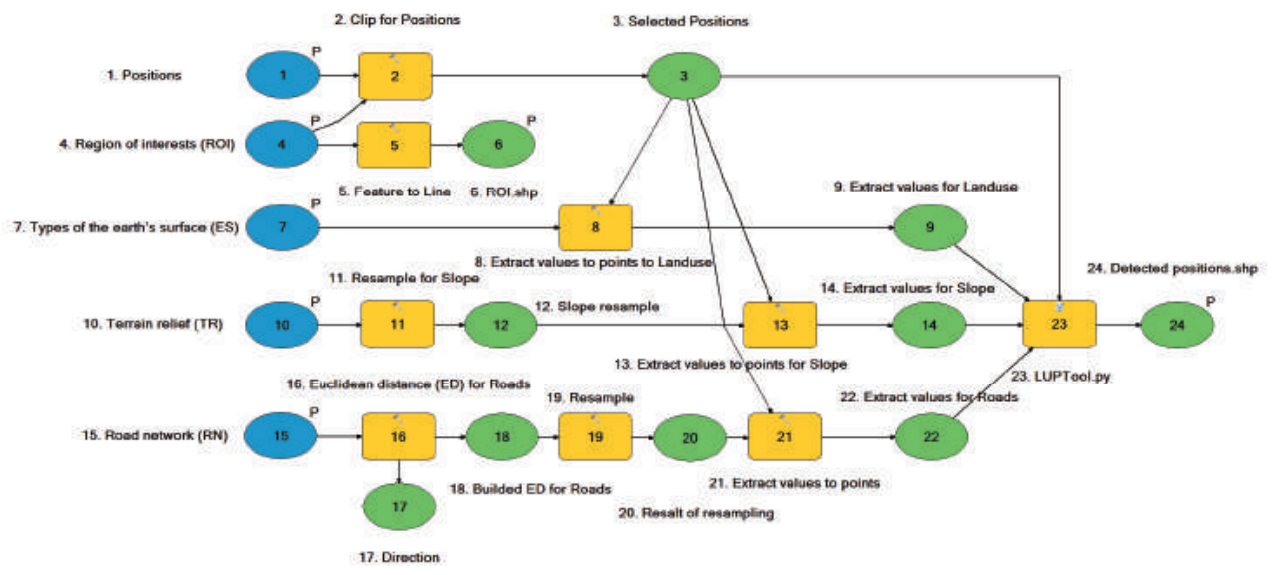

Fig. 4 LUP\&ThreeSupplementOpinions tool's schema in the ArcGIS ModelBuilder

At the process of modeling, the decision on the class of any object $X_{q}$ was being formed as follows. If the opinion of the O-I regarding the class of the $X_{q}$ object had a relatively small uncertainty, namely: inequality $\operatorname{PIC}\left[E^{\text {cons }}\left(X_{q}\right)\right] \geq 0.5$ was held, then the passage to the block 10 was directly carried out, where the final decision on the object class was made in accordance with the Eq. (39).

If the opinion of the O-I regarding the class of the $X_{q}$ object had a significant uncertainty $\left(P I C\left[E^{\text {cons }}\left(X_{q}\right)\right]<0.5\right.$ case $)$, the request into the database was being generated in order to obtain a new portion of the needed geospatial information (this is the corresponding digital terrain map layer), and after that the revised opinion was being formed.

Three variants of the classification were being studied:

- (V/1) when the final decision on the class of the object was made attracting contextual information about the ES only ( $G=1$ case),

- $(\mathrm{V} / 2)$ when the final decision on the class of the object was made attracting contextual information about the ES and the TR ( $G=2$ case),

- (V/3) when the final decision on the class of the object was made attracting contextual information about the ES, the TR, and the RN ( $G=3$ case). 


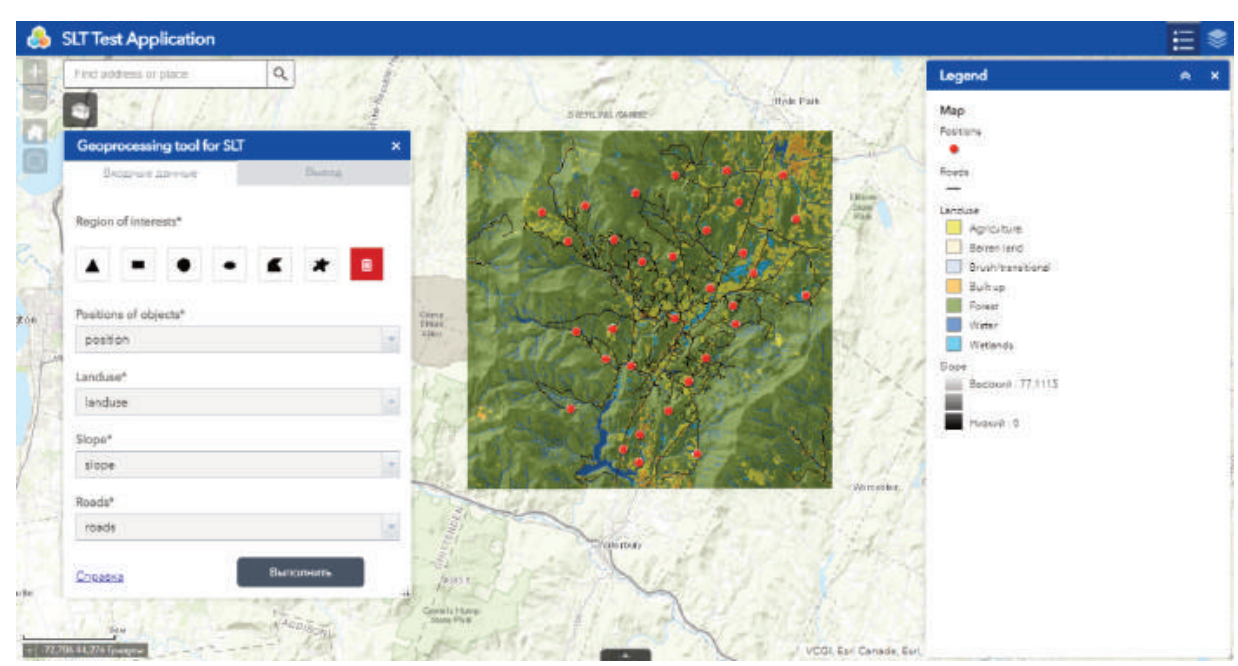

Fig. 5 Interface of geoportal with geoprocessing tool

The interface of the geoportal with the results of the object classification for last case (V/3) is shown in Fig. 6.

The results of modeling (Tab. 2) confirm the validity of the idea that increasing the amount of contextual geospatial information incorporated into the classification process allow appreciably to improve the classification accuracy. So, if the accuracy of cognitive classification (the visual analysis result of the multispectral image by the OI, i.e. V/0 variant) was 0.65 , then the involvement of contextual information about the ES only (V/1 variant) made it possible to increase the accuracy to 0.78 ; and the joint attracting contextual information about the ES, the TR, and the RN (V/3 variant) provided an increase in accuracy up to 0.93 .

Comparison of the results of cognitive classification (V/0 variant) and classification with attracting the geospatial context (V/3 variant) shows that the probability of correct classification was increased by 1.43 times.

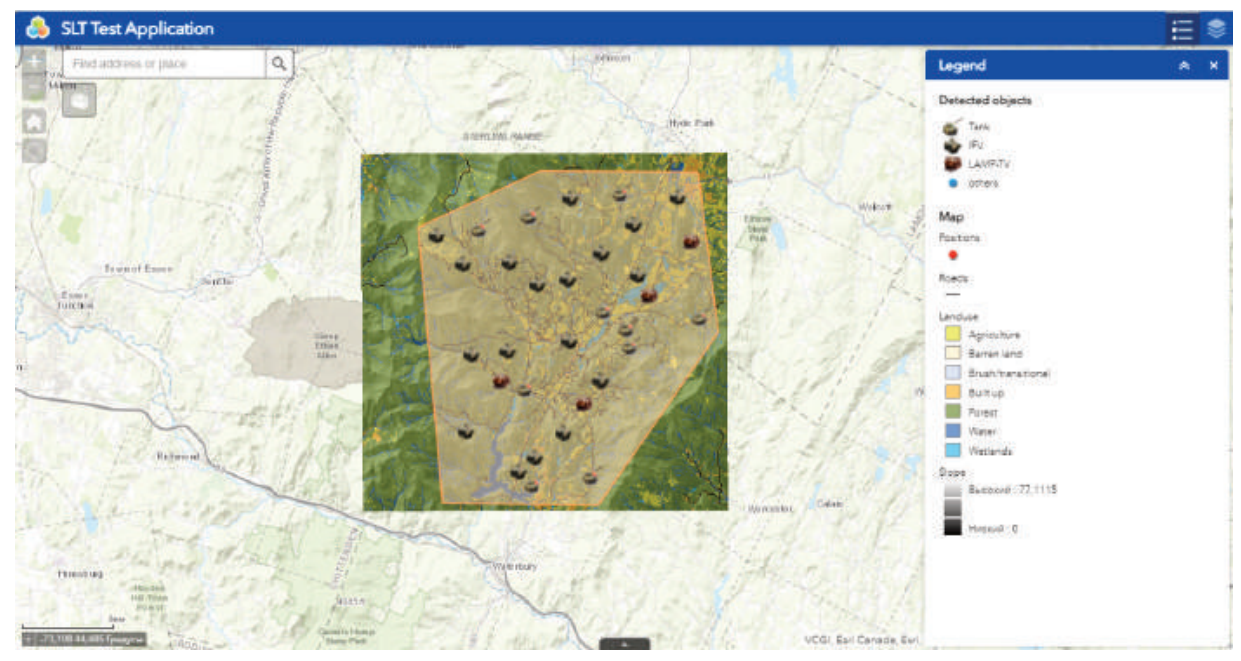

Fig. 6 Interface of geoportal with classified objects 
Tab. 2 Probabilities of correct classification of objects

\begin{tabular}{|c|c|c|c|}
\hline Variant & $\begin{array}{c}\text { Threshold } \\
G_{\text {thd }}\end{array}$ & $\begin{array}{c}\text { Attracted context geospatial } \\
\text { information }\end{array}$ & $\begin{array}{c}\text { Probability of cor- } \\
\text { rect classification }\end{array}$ \\
\hline $\mathrm{V} / 0$ & 0 & without geospatial information & 0.65 \\
\hline $\mathrm{V} / 1$ & 1 & ES & 0.78 \\
\hline $\mathrm{V} / 2$ & 2 & ES \& TR & 0.84 \\
\hline $\mathrm{V} / 3$ & 3 & ES \& TR \& RN & 0.93 \\
\hline
\end{tabular}

\section{Discussion}

Today's means of intelligence data acquisition make it possible to obtain huge amounts of information, but the processing of this information is a serious problem. Therefore, analysts are assisted by computerized systems of information support and decision-making [34]. The most advanced systems of this kind include Disciple-EBR [35] and GEOART [36].

The Disciple-EBR system helps the analyst perform the calculations necessary for solving such issues as information retrieval, evidence checking and argumentation, hypothesis validation, etc. The GEOART is a tool for the semiautomated analysis of geospatial data (on the base of the artificial intelligence techniques) and the geoinformation products generation.

However, both the named and other known similar systems are designed to help the O-I under strictly defined scenarios and classes of objects of interest, without taking into account the model of human decision-making; besides, the interaction of the human and the computer system is situational. Therefore, the creation of new methods and systems for the information support of the O-I is a topical issue.

Our method for computer-aided analysis of aerial and satellite multispectral images is largely free of the above disadvantages. Since the primary classification is carried out by the O-I, then there is an opportunity to work with a wide variety of ground scenes and an open nomenclature of objects of interest. The method allows to correct (mitigate) possible cognitive errors of the O-I, and all operators classification decisions go through the computer.

A certain disadvantage of the developed method is that when deciding on the class of the object in question, information about the presence, class and location of other objects of interest is not taken into account. In principle, such information could be useful in complex classification cases.

\section{Conclusion}

In the paper, the new method to improve the classification accuracy of objects in digital multispectral images is proposed. The core idea of the research is to increase the accuracy of object classification in aerial and satellite images via incorporating context in the form of geospatial information about the terrain into the classification process. The idea is implemented with help of the multi-step procedural scheme. As a mathematical basis of the developed method, the authors used SL of A. Jøsang.

The efficiency of the proposed method was assessed with help of computer modeling. For this purpose, the special SLTToolbox toolkit was developed in the ArcGIS 
ModelBuilder. The basic element of the toolkit is the script LUPTool.py in Python. The results of modeling have demonstrated the effectiveness of the proposed method.

Further work of the authors is expected to be aimed at simplifying the procedure for searching for geospatial information necessary to make a final decision on the class of each object under consideration. It will also be interesting to develop more efficient algorithms for correcting (mitigating) typical cognitive errors of OI.

\section{References}

[1] WANG, G. and Q. WENG. Remote Sensing of Natural Resources. New York: CRC Press, 2014. ISBN 978-1-4665-5692-8.

[2] MCRP 2-10B.5, Imagery Intelligence [online]. 2016 [viewed 2021-10-08]. Available from: https://www.marines.mil/portals/1/Publications/MCRP\%20210B.5\%20GN.pdf?ver=2019-02-07-120201-527

[3] HANNEVIK, T.N.A., K. ELDHUSETET, Ø.K. LENSJØ, D.J. WEYDAHL, R.B. OLSEN, B. van den BROEK, R. DEKKERAL, M. VAN PERSIE, H. NOORBERGEN, A. OOSTDIJK and R. VAN SWOL. Military Use of Space. WP1 Space-Based Geospatial Intelligence [online]. Norwegian Defense Research Establishment, 2017 [viewed 2021-10-15]. ISBN 978-8-2464-2993-9. Available from: https://publications.ffi.no/nb/item/asset/dspace:4251/17-16935.pdf

[4] CLARK, M.R. Geospatial Intelligence: Origins and Evolution. Washington: Georgetown University Press, 2020. ISBN 978-1-64712-011-5.

[5] JOSEPH, G. Building Earth Observation Cameras. New York: CRC Press, 2015. ISBN 978-1-4665-6647-7.

[6] RACEK, F., T. BALÁŽ and P. MELŠA. Hyperspectral Data Conversion in the Case of Military Surveillance. Advances in Military Technology, 2015, 10(1), pp. 5-13. ISSN 1802-2308.

[7] LILlESAND, T.M., R.W. KIEFER and J.W. CHIPMAN. Remote Sensing and Image Interpretation. $7^{\text {th }}$ ed. Hoboken: Wiley, 2015. ISBN 978-1-1183-4328-9.

[8] HEUER, Jr. R.J. and R.H. PHERSON. Structured Analytic Techniques for Intelligence Analysis. New York: CQ Press, 2011. ISBN 978-1-60871-018-1.

[9] GADEPALLY, V.N., B.J. HANCOCK, K.B. GREENFIELD, J.P. CAMPBELL, W.M. CAMPBELL and A.I. REUTHER. Recommender Systems for the Department of Defense and Intelligence Community. Lincoln Laboratory Journal, 2016, 22(1), pp. 74-89. ISSN 0896-4130.

[10] BRYNIELSSON, J., A. HORNDAHL, L. KAATI, C. MÅRTENSON and P. SVENSON. Development of Computerized Support Tools for Intelligence Work. In: Proceedings of the $14^{\text {th }}$ International Command and Control Research and Technology Symposium [online]. 2009 [viewed 2021-10-01]. Available from: https://pdfs.semanticscholar.org/7fd7/143e9c1d443818d58880f4b12d85b83ed0fe.pdf

[11] TECUCI, G., L. KAISER, D. MARCU, C. UTTAMSINGH and M. BOICUET. Evidence-based Reasoning in Intelligence Analysis: Structured Methodology and System. Computing in Science and Engineering, 2018, 20(6), pp. 9-21. DOI 10.1109/MCSE.2018.2873852. 
[12] New Research Directions for the National Geospatial-Intelligence Agency: Workshop Report. Washington: National Academies Press, 2010. ISBN 978-03091-5865-7.

[13] BRÉZILLON, P. Context in Problem Solving: A Survey. The Knowledge Engineering Review, 1999, 14(1), pp. 47-80. DOI 10.1017/S0269888999141018.

[14] ROGOVA, G.L. and A.N. STEINBERG. Formalization of "Context" for Information Fusion. In: L. SNIDARO, J. GARCÍA, J. LLINAS and E. BLASCH, eds. Context-Enhanced Information Fusion. Cham: Springer, 2016, pp. 27-43. DOI 10.1007/978-3-319-28971-7_2.

[15] CONTAT, M., V. NIMIER and R. REYNAUD. Request Management Using Contextual Information for Classification. In: Proceedings of the $5^{\text {th }}$ International Conference on Information Fusion. FUSION 2002. Annapolis: IEEE, 2002, pp. 1147-1153. DOI 10.1109/ICIF.2002.1020942.

[16] CONDESSA, F., J. BIOUCAS-DIAS, C. CASTRO, J. OZOLEK and J. KOVACEVIC. Classification with Rejection Option Using Contextual Information. In: 2013 IEEE $10^{\text {th }}$ International Symposium on Biomedical Imaging. San Francisco: IEEE, 2013, pp. 1340-1343. DOI 10.1109/ISBI.2013.6556780.

[17] CAYUELA, L., J.D. GOLICHER, J.S. REY and J.M.R. BENAYAS. Classification of a Complex Landscape Using Dempster-Shafer Theory of Evidence. International Journal of Remote Sensing, 2006, 27(10), pp. 1951-1971. DOI 10.1080/01431160500181788.

[18] MOMANI, B.A., P. MORROW and S. MCCLEAN. Fusion of Elevation Data into Satellite Image Classification Using Refined Production Rules. In: M. KAMEL and A. CAMPILHO, eds. Image Analysis and Recognition. ICIAR 2011. Lecture Notes in Computer Science. Heidelberg: Springer, 2011, pp. 211220. DOI 10.1007/978-3-642-21593-3_22.

[19] SHAFER, G. A Mathematical Theory of Evidence. Princeton: Princeton University Press, 1976. ISBN 978-0-6911-0042-5.

[20] ALLEN, D.W. Getting to Know ArcGIS: ModelBuilder. Redlands: ESRI Press, 2011. ISBN 978-1-5894-8255-5.

[21] OBERKAMPF, W.L., S.M. DELAND, B.M. RUTHERFORD, K.V. DIEGERT and K.F. ALVIN. Error and Uncertainty in Modeling and Simulation. Reliability Engineering and System Safety, 2002, 75(3), pp. 333-357. DOI 10.1016/S09518320(01)00120-X.

[22] WHITE, R.A., A. ÇÖLTEKIN and R.R. HOFFMAN. Remote Sensing and Cognition: Human Factors in Image Interpretation. Boca Raton: CRC Press, 2018. ISBN 978-1-3510-4046-4.

[23] DEVITT, S.K., T.R. PEARCE, T. PEREZ and P.D. BRUZA. Mitigating against Cognitive Bias when Eliciting Expert Intuitions. In: International Conference on Thinking [online]. Brisbane: Queensland University of Technology, 2016, pp. 1-6 [viewed 2020-10-01]. Available from: https://eprints.qut.edu.au/98542/1/ Cognitive_bias_expert_options_Handout_20160802_skd.pdf

[24] PRAVA, V. Identifying and Correcting Cognitive Biases in Subjective Probability Elicitation Surveys: Model Based Approaches [online]. Baltimore: Johns Hopkins University, 2016 [viewed 2020-10-01]. Available from: https://jscholar 
ship.library.jhu.edu/bitstream/handle/1774.2/39698/PRAVA-DISSERTATION2016.pdf

[25] ZADEH, L.A. On the Validity of Dempster's Rule of Combination [online]. Berkeley: University of California, 1979 [viewed 2020-10-01]. Available from: http://www2.eecs.berkeley.edu/Pubs/TechRpts/1979/ERL-m-79-24.pdf

[26] JØSANG, A. Subjective Logic: A Formalism for Reasoning under Uncertainty. Cham: Springer, 2016. ISBN 978-3-3194-2337-1.

[27] POPOV, M.O., M.V. TOPOLNYTSKYI, O.V. TITARENKO, S.A. STANKEVICH and A.A. ANDREIEV. Forecasting Gas and Oil Potential of Subsoil Plots via Co-analysis of Satellite, Geological, Geophysical and Geochemical Information by Means of Subjective Logic. WSEAS Transactions on Computer Research, 2020, 8, pp. 90-101. DOI 10.37394/232018.2020.8.11.

[28] SMETS, P. and R. KENNES. The Transferable Belief Model. Artificial Intelligence, 1994, 66(2), pp. 191-234. DOI 10.1016/0004-3702(94)90026-4.

[29] JØSANG, A. The Consensus Operator for Combining Beliefs. Artificial Intelligence Journal, 2002, 142(1-2), pp. 157-170. DOI 10.1016/S00043702(02)00259-X.

[30] DI, L. and B. KOBLER. NASA Standards for Earth Remote Sensing Data. In: International Archives of Photogrammetry and Remote Sensing [online]. Amsterdam: ISPRS, 2000, pp. 147-155 [viewed 2020-10-01]. Available from: https://www.isprs.org/proceedings/XXXIII/congress/part2/147_XXXIII-part2.pdf

[31] DEZERT, J., D. HAN, Z. LIU and J.-M. TACNET. Hierarchical DSmP Transformation for Decision-Making under Uncertainty. In: $201215^{\text {th }}$ International Conference on Information Fusion. Singapore: IEEE, 2012, pp. 294-301. ISBN 978-1-4673-0417-7.

[32] JØSANG, A. A Logic for Uncertain Probabilities. International Journal of Uncertainty, Fuzziness and Knowledge-Based Systems, 2001, 9(3), pp. 279-311. DOI 10.1142/S0218488501000831.

[33] SUDANO, J.J. Pignistic Probability Transforms for Mixes of Low- and HighProbability Events. In: Proceedings of $4^{\text {th }}$ International Conference on Information Fusion [online]. Montreal: Inffus, 2001, pp. 23-27 [viewed 2020-10-01]. Available from: https://arxiv.org/ftp/arxiv/papers/1505/1505.07751.pdf

[34] BOSCH, K. van den and A. BRONKHORST. Human-AI Cooperation to Benefit Military Decision Making. In: Proceedings of the NATO IST-160 Specialist meeting on Big Data and Artificial Intelligence for Military Decision Making [online]. Bordeaux: S\&T, 2018, pp. S 1-13 [viewed 2021-11-09]. Available from: https://www.sto.nato.int/publications/STO\%20Meeting\%20Proceedings/STOMP-IST-160/MP-IST-160-S3-1.pdf

[35] TECUCI, G., S. MECKL, D. MARCU and M. BOICU. Instructable Cognitive Agents for Autonomous Evidence-Based Reasoning. Advances in Cognitive Systems, 2019, 8, pp. 73-92. ISSN 2324-8416.

[36] LANCE, M., D.A. GOLDFELD, A. TINGSTAD, S. LINGEL and E. GEIST. Technology Innovation and the Future of Air Force Intelligence Analysis: Volume 1, Findings and Recommendations. Santa Monica: RAND Corporation, 2021. ISBN 978-1-9774-0631-6. 